\title{
Forecasting The South African Business Cycle Using Fourier Analysis
}

\author{
Daniel Thomson, North-West University, South Africa
}

Gary van Vuuren, North-West University, South Africa

\begin{abstract}
A Fourier transform analysis is proposed to determine the duration of the South African business cycle, measured using log changes in nominal gross domestic product (GDP). The most prominent cycle (two smaller, but significant, cycles are also present in the time series) is found to be 7.1 years, confirmed using Empirical Mode Decomposition. The three dominant cycles are used to estimate a 3.5 year forecast of log monthly nominal GDP and these forecasts compared to observed (historical) data. Promising forecast potential is found with this significantly-reduced number of cycle components than embedded in the original series. Fourier analysis is effective in estimating the length of the business cycle, as well as in determining the current position (phase) of the economy in the business cycle.
\end{abstract}

Keywords: Fourier; Hodrick Prescott Filter; Baxter-King Filter; Empirical Mode Decomposition

\section{INTRODUCTION}

$\mathrm{n}$ finance and economics, the predominant method of analysing time-series data is usually to view these
data in the time-domain, i.e., analysing changes of a series as it progresses through time. The problem in using only this approach to study financial datasets is that all realisations are recorded at a predetermined frequency. This frequency corresponds to whichever period the realisations are recorded at and the implicit assumption is made that the relevant frequency to study the behaviour of the variable matches with its sampling frequency (Masset, 2008). This can be construed as analysing inflation figures with a one year time frame and presuming that the cycle will repeat itself the following year as the cycle is presumed to be one year long. The realisations of financial and economic variables often depend on a number of frequency components rather than just one so the time-domain approach alone will not be able to process the information in the time-series precisely (Masset, 2008).

Spectral analysis methods that enable a frequency-domain representation of the data, such as Fourier series and wavelet methods, are able to identify at which frequencies the time series variable is active. The strength of the activity may be measured using Fourier analysis to construct a frequency spectrum (or periodogram) ${ }^{1}$ - a graphic representation of the intensity of a frequency component potted against the frequency at which it occurs. This method is particularly attractive for the use of economic variables that exhibit cyclical behaviour as the cycle length may be identified using the Fourier transform (Baxter \& King, 1999).

Understanding the business cycle of a region and having an idea of its current position (or phase in the cycle) enables participants in the economy to make informed decisions. Because business cycle information is so valuable, much research has been done to identify its behaviour and the South African business cycle is no exception (see Venter (2005), Bosch and Ruch (2012) and Du Plessis et. al (2014)). In fact, owing to South Africa's volatile political and economic history, modelling its behaviour provides a robust test to structural breaks and regime shifts of any technique (Aaron \& Muellbauer, 2002 and Chevillon, 2009).

This article examines and attempts to forecast South African Gross Domestic Product (GDP) time-series data using Fourier series analysis. Significant cycles are detected and the length of these cycles quantified by examining the data in the frequency-domain rather than the time-domain, thus providing an alternative method of business cycle analysis to most in the literature. Using single frequency components or a combination thereof also provides a novel perspective

${ }^{1}$ The terms frequency spectrum and periodogram are used interchangeably. 
of South African GDP forecasting. A further aim of this work is that it paves the way for further, alternative methods of analysis of economic and financial variables, wavelets in particular, through highlighting potential limitations of pure frequency-domain analysis.

The remainder of this paper is structured as follows. Section 2 provides a brief literature review providing an overview of spectral methods applied to finance and previous attempts at modelling South African GDP. A description of the data used in the analysis as well as detail of methodologies employed is given in Section 3. The analysis, results and discussion of the problem are presented in Section 4. Section 5 concludes and provides recommendations for further study.

\section{LITERATURE REVIEW}

Spectral analysis methods have a broad range of applications in the real world. The Fourier integral formula - which enjoys widespread physics and engineering applications - is regarded as one of the most fundamental results of modern mathematical analysis (Debnath, 2012), including spectroscopy, crystallography, imaging, signal processing and communications. Fourier series have more gained traction as a tool in finance and econometrics, starting with the early works of Cunnyngham (1963), Nerlove (1964) and Granger (1966), and others on simple economic time series in the 1960 s to modern day applications in derivative pricing and wavelet analysis.

This literature review focuses on the application to finance and econometrics. In particular, literature that describes practical uses of Fourier analysis as applied to modelling and forecasting economic data is presented.

Hamilton (1994) discussed spectral analysis and introduced the concepts of the population spectrum, the sample periodogram and estimation based on the population spectrum. ${ }^{2}$ An analysis of US manufacturing data demonstrated the use of spectral methods on real time series. Hamilton (1994) explains why adjustment (in the form of taking natural logs) of the data must be performed, owing to the assumptions of a covariance-stationary process implicit in the transform (for a mathematical definition of a covariance-stationary process, see Lindgren, Rootzén \& Sandsten (2013)). To omit seasonal effects which appeared in the periodogram, (Hamilton, 1994) suggested using year-on-year growth rates.

General and theoretical work on spectral analysis in financial time series was introduced by Cunnyngham (1963), Granger \& Hatanaka (1964) and Granger (1966). Shumway \& Stoffer (2000) provide a wide range of time series analysis techniques and applications, covering spectral analysis and filtering. Granger \& Morgenstern (1964) used Fourier analysis to examine stock market prices on New York Stock Exchange price series.

Granger \& Morgenstern (1964) found that stock prices followed the random-walk hypothesis in the short term, but long run components exerted greater influence than the random-walk hypothesis suggested (a non-parametric test of this was demonstrated by a flat spectrum of share price changes). Seasonal variation and the business-cycle components were found to be largely irrelevant in explaining the evolution of stock market prices.

Praetz (1973) studied Australian share prices and share price indices in the frequency domain using spectral analysis methods. In contrast to the findings of Granger \& Morgenstern (1964), they found small departures from the randomwalk hypothesis in their share price series from 1947 to 1968, although not large enough to abandon the hypothesis as a crude first approximation. Some clearly-defined seasonal patterns were present. Praetz's (1973) study of share price indices and other market sectors were shown to lead or lag the market as a whole. This evidence contrasted with Granger and Morgenstern's (1964) and Godfrey, Granger \& Morgenstern's (1964) studies on the New York Stock Exchange and the London Stock Exchange, where such patterns and lags were far less significant. Praetz (1973) offered the tentative conclusion that Australian share markets were less efficient than their overseas counterparts.

Iacobucci (2003) elaborated on the issues of cross-spectral analysis and filtering, and explored the concepts of coherency and phase spectra. This analysis was applied to US inflation and unemployment data and showed that a Phillips relation existed at typical business cycle components of 14 and six years. Cross-spectral analysis and filtering

\footnotetext{
${ }^{2}$ Spectral analysis meaning the study of a variable over the frequency spectrum or frequency-domain.
} 
was used to measure the correlation between the two factors through the Phillips curve. Unemployment was found to lead inflation by a full 12 months.

Masset (2008) provided an introduction to spectral and wavelet methods of analysis with many practical examples using real economic data. A spectral analysis on residential property prices in New York covering the period January 1987 to May 2008 was performed using both parametric and non-parametric methods to show the subsequent differences in the frequency spectrum. Spectra from the non-parametric methods (using Welch method (Welch, 1967) contained more noise than the spectra obtained from the parametric methods (Yule-Walker and Burg (Tjostheim \& Paulsen, 1983). Fourier analysis of the data confirmed that strong seasonalities affected New York home prices with a cycle of 12 months. A detailed exposition on wavelet analysis was proffered as a way to overcome many of the shortfalls of Fourier transform and filtering methods (Masset, 2008).

Liu et al., (2012) investigated business and growth cycles in the frequency domain by performing Fourier analyses on several data sources including electricity demand, foreign currency data, monthly retail sales, quarterly GDP, labour market and productivity statistics from Statistics New Zealand. In their analysis of the GDP data, the data were transformed using natural logarithms and detrended using the Hodrick-Prescott filter before conducting Fourier analysis of the detrended transformed data. Using a periodogram, definitive cycles corresponding to eight years and four-and-a-half years were found. Because of the distance between energy spikes in the periodogram, it was proposed that the cycle length varied between 4.5 to 8 years. Liu et al., (2012) argued that Fourier analysis could be used to detect cyclical behaviour in any type of time series data, although no cyclical behaviour was detected in the majority of the time series data tested and proposed that a natural extension to Fourier analysis was the use of wavelets.

Omekara, Ekpenyong \& Ekrete (2013) used Fourier series analysis to identify cycles in the Nigerian all-items monthly inflation rates from 2003 to 2011. A square root transformation was used to increase stability and normality of the inflation rate data. Periodogram analysis showed a short term and a long term cycle of 20 months and 51 months respectively with the long cycle corresponding to the length of two different government administrations that existed during the sample period. A general Fourier series model was then fitted to the data to make accurate short term monthly inflation rate forecasts from an out-of-sample period from September 2011 to September 2012.

More recent academic research (Masset, 2008 and Liu et al., 2012) of Fourier series often leads to a recommendation of wavelet analysis as a natural extension to the limited, frequency-domain only methods such as Fourier transforms. Masset (2008) presents two principal drawbacks to the use of spectral analysis and standard filtering methods:

(i) they impose strong restrictions regarding the possible processes underlying the dynamics of the series (e.g. stationarity), and,

(ii) they lead to a pure frequency-domain representation of the data, i.e. all information from the time-domain representation is lost in the operation.

Much literature governing spectral analysis now embraces wavelets, with Fourier analysis forming part of the development process (see, for example, Daubechies, 1988; Vetterli \& Herley, 1992; Scargle, 1993 \& Cody, 1994).

Aron \& Muellbauer (2002) developed a GDP forecasting model for South Africa to measure interest rate effects on output. Multistep forecasting models were found to be preferable to recursive forecasting with vector autoregressive (VAR) models because of the structural breaks present in the South African economy. The multistep model comprised a factor model which was evolved to a single equation equilibrium correction model with a built in term for the stochastic trend. The model made forecasts for up to four quarters and was tested for stability using sample breaks. Tests for normality and heteroscedasticity yielded satisfactory, robust results (Aron \& Muellbauer, 2002).

Venter (2005) discusses the methodology used by the South African Reserve Bank (SARB) to identify business cycle turning points. This methodology included the use of three composite business cycle indicators and two diffusion indexes. Leading, lagging and coincident indicators make up the composites while movement in historic and current diffusion indexes helped to confirm whether changes in the economy were localised or all-encompassing. 
Chevillon (2009) drew on the research of Aron \& Muellbauer (2002) and established whether direct multi-step estimation improved the accuracy of forecasts. Chevillon (2009) assembled 779 different models and applied them to South African GDP data to ascertain which provided the most accurate forecasts and coped best with the large number of regime changes and structural breaks. Aron \& Muellbauer's (2002) direct multi-step model was found to perform best within short time horizons whilst multivariate and univariate models performed better with intermediate to long term time horizons.

Bosch \& Ruch (2012) provided an alternative methodology to dating business cycle turning points in South Africa by using a Markov switching model and the Bry-Boschan method to date the turning points and found that the model estimates generally coincided with the business cycle turning points determined by the SARB. The model was applied to GDP data, but also to 114 of the 186 stationary variables the SARB uses to date the business cycle (Bosch and Ruch, 2012). Using Principle Component Analysis (PCA) on these variables a more accurate measure of the business cycle turning points than using GDP data alone was obtained.

Du Plessis, Smit \& Steinbach (2014) developed a dynamic stochastic general equilibrium (DSGE) model for the South African economy. The model used Bayesian techniques to incorporate prior information about the economy into the parameter estimates. Its forecasting capability extends up to seven quarters and was tested against a panel of professional forecasters and a random walk. It was found to outperform the professional forecasters and the random walk, especially over longer time horizons, when used to predict CPI inflation and GDP growth.

Other filters exist - such as the Kalman filter - which may be used to extract underlying patterns (including periodic ones) from signal data by reducing or eliminating noise. To calibrate the Kalman filter, however, requires many data and most GDP data are only reported and recorded quarterly. Considerably more data are required for the filter to 'learn' the underlying pattern (i.e. distinguish the coherent pattern from the noisy signal) as well as test out of sample data than are needed for use in, e.g. the Fourier transform technique. For this reason, the Kalman filter was not used in this work.

\section{DATA AND METHODOLOGY}

\subsection{Data}

This study seeks a simple and readily available proxy for South African economic activity from which to identify potentially meaningful cycles. GDP, although not a perfect measure of the business cycle (see Boehm \& Summers, 1999), provides a reasonable measure of economic activity and the business cycle, over a satisfactory sample period.

The data employed are seasonally-adjusted, nominal GDP in South African rand (ZAR) measured monthly from January 1969 to July 2015. The in-sample period for the establishment of dominant cycles using Fourier analysis was January 1969 to December 2011 and the out-of-sample forecast period was January 2012 to July 2015 (SARB, 2015). Fourier analysis uses a Fast Fourier Transform (FFT) which enables much faster computing, but restricts the number of data points to $2^{n}$, where $n \in \mathbb{Z}$ (Cooley and Tukey, 1965).

\subsection{Methodology}

The Hodrick-Prescott (HP) and Baxter-King (BK) filters are time-domain techniques to decompose time-series data into trend and cycle components. The cycle component of the data may then be analysed using methodologies which require stationarity, such as the FFT.

\subsubsection{Time Series Filtering Methods}

\section{Hodrick-Prescott Filter}

Hodrick \& Prescott (1997) presented a procedure for representing a time series $X_{t}$ as the sum of a smoothly-varying trend component $\tau_{t}$, and a cyclical component $c_{t}$, 
where,

$$
X_{t}=\tau_{t}+c_{t} \quad t=1,2, \ldots T
$$

The trend component $\tau_{t}$ is found by choosing a positive value of $\lambda$ and solving for

$$
\min \left\{\sum_{t=1}^{T}\left(y_{t}-\tau_{t}\right)^{2}+\lambda \sum_{t=2}^{T}\left[\left(\tau_{t+1}-\tau_{t}\right)-\left(\tau_{t}-\tau_{t-1}\right)^{2}\right]\right\} .
$$

The parameter $\lambda$ is a smoothing parameter which penalises variability in the growth (trend) component series (Hodrick $\&$ Prescott, 1997). The larger the value of $\lambda$, the smoother is the output series.

The HP filter has been criticised for a number of limitations and undesirable properties. Canova (1994 and 1998) used the HP filter to extract business cycles from macroeconomic data of average length of four to six years, but was sceptical of the methodology used to determine key parameter inputs after inconsistent results were obtained. Spurious cycles and distorted estimates of the cyclical component when using the HP filter were obtained by Harvey and Jaeger (1993). Cogley \& Nason (1995) also found spurious cycles when using the HP filter on difference-stationary input data. Application of the HP filter to US time series data was found to alter measures of persistence, variability and comovement dramatically (King \& Rebelo, 1993). Many of these critiques do not provide sufficient compelling evidence to discourage use of the HP filter (van Vuuren, 2012). As a result, it remains widely-used among macroeconomists for detrending data which exhibit short term fluctuations superimposed on business cycle-like trends (Ravn \& Uhlig, 2002).

\section{BK Filter}

Baxter and King (1999) argued that a time series function $X_{t}$ comprised three components: a trend $\tau$, a cyclical component $c$, and a 'noise' (random) component, $\epsilon$, such that:

$$
X_{t}=\tau_{t}+c_{t}+\epsilon_{t} \quad t=1,2, \ldots T
$$

The BK filter removes the trend and noise components, leaving only the cycle component:

$$
c_{t}=X_{t}-\tau_{t}-\epsilon_{t} \quad t=1,2, \ldots, T .
$$

Guay \& St-Amant (2005) provided a measure of the ability of the HP and BK filters to extract the business cycle component of macroeconomic time series using two different business cycle definitions. The first definition was that the duration of a business cycle was between six and 32 quarters (as argued by Baxter \& King (1999). The second definition made a distinction between permanent and transitory components. Guay \& St-Amant (2005) concluded that in both cases the filters performed adequately if the spectrum of the original series peaked at business-cycle frequencies. Low frequencies dominant in the spectrum were found to distort the business cycle. Guay \& St-Amant's (2005) results suggest that the use of HP and BK filters on series resembling the Granger shape of an economic variable may be problematic.

In this work, Fourier analysis was performed on the cyclical components of the HP filter (1) and the BK filter (2), for comparison.

\subsubsection{Data Stationarity}

A practical difficulty arises when the deterministic time-varying component (drift) of the underlying data is timevarying. How may this deterministic component be removed and the random process component be isolated? The drift may be thought of as the local mean value at each point in time, while the variation around this means represents the signal. Removing this drift may be accomplished through several techniques - two are considered below. 


\section{Log of Time Series}

Masset (2009) emphasises that spectral methods such as Fourier transforms require that data under investigation must be stationary (for a comprehensive definition of strict stationarity and weak stationarity, see Pelagatti (2013) who takes stationarity to mean weak stationarity (covariance-stationary), unless otherwise specified). An augmented Dickey-Fuller (ADF) test was used to check for this condition. The results obtained from the ADF test on nominal GDP data used failed to reject the null hypothesis that the index levels series is non-stationary. Natural logarithms of the time-series were thus taken $\left(\ln \left(x_{t}\right)-\ln \left(x_{t-1}\right)\right)$, where $x_{t}$ and $x_{t-1}$ are consecutive months in the GDP time series. This transformation converts the monthly nominal GDP data into monthly returns, a stationary series.

\section{Empirical Mode Decomposition (EMD)}

The EMD is specifically designed to expand a function into a trend plus a number of intrinsic mode functions (IMFs), whose defining features are a) that all local minima and maxima are negative and positive, respectively, and $b$ ) that their local mean is zero. To apply the EMD, the envelope of a signal $x(t)$ may be generated by first determining the sequences $H=\left\{h_{i}\right\}_{i \in \mathbb{Z}}$ and $L=\left\{l_{i}\right\}_{i \in \mathbb{Z}}$ i.e. the local maxima and minima of $x$, respectively. Let $\operatorname{Lin}_{A, B}(t)$ be the continuous, linearly interpolated function assuming the values $B=\left\{b_{i}\right\}_{i \in \mathbb{Z}}$ on $A=\left\{a_{i}\right\}_{i \in \mathbb{Z}}$ and changing slope on $A$ only. Let $x^{\uparrow}$ be the pointwise maximum between $x$ and $\operatorname{Lin}_{H, x(H)}(t)$ and $x^{\downarrow}$ the pointwise minimum between $x$ and $\operatorname{Lin}_{L, x(L)}(t)$. The pair $x^{\uparrow}, x^{\downarrow}$ forms the envelope of $x\left(x^{\downarrow}(t) \leq x(t) \leq x^{\uparrow}(t)\right)$ and the drift is:

$$
\bar{x}(t)=\frac{\left(x^{\uparrow}(t)+x^{\downarrow}(t)\right)}{2}
$$

$x_{1}=x-\bar{x}$ is the oscillating component, and this method may be applied multiple times to obtain $x_{1}=\bar{x}_{1}+x_{2}$, etc. Higher order splines (such as cubic splines) are applied at this point to generate smoother envelopes than those generated from piecewise linear splines (Huang, Shen, Long, et al, 1998).

\subsubsection{Fourier Analysis}

The central idea of spectral analysis is to re-express the original time-series $x(t)$ as a new sequence $X(f)$, which evaluates the significance of each frequency component, $f$, in the dynamics of the original series (Masset, 2008). This is achieved by using the discrete version of the Fourier transform, which decomposes a periodic signal into its constituent frequencies. Time series data that comprise periodic components can be written as a sum of simple waves (that is, oscillations of a single frequency) represented by sine and cosine functions (Brown and Churchill, 1993). A Fourier series is an expansion of a periodic function in terms of an infinite sum of sines and cosines by making use of the orthogonality relationships of the sine and cosine functions (Askey \& Haimo, 1996). The generalised Fourier series, obtained using the functions $f_{1}(x)=\cos x$ and $f_{2}(x)=\sin x$ (which form a complete orthogonal system over $[-\pi, \pi])$ gives the Fourier series of a function $f(x)$ :

$$
f(x)=\frac{1}{2} a_{0}+\sum_{n=1}^{\infty} a_{n} \cos (n x)+\sum_{n=1}^{\infty} b_{n} \sin (n x)
$$

where

$$
\begin{aligned}
& a_{0}=\frac{1}{\pi} \int_{-\pi}^{\pi} f(x) d x \\
& a_{n}=\frac{1}{\pi} \int_{-\pi}^{\pi} f(x) \cos (n x) d x \text { and } \\
& b_{n}=\frac{1}{\pi} \int_{-\pi}^{\pi} f(x) \sin (n x) d x .
\end{aligned}
$$


For a function $f(x)$ periodic on an interval $[0,2 L]$ instead of $[-\pi, \pi]$, a simple change of variables may be used to transform the interval of integration from $[-\pi, \pi]$ to $[0,2 L]$ by letting

$$
x=\frac{\pi x^{\prime}}{L}
$$

Solving for $x^{\prime}$ and substituting into (3) gives (Krantz, 1999):

$$
f\left(x^{\prime}\right)=\frac{1}{2} a_{0}+\sum_{n=1}^{\infty} a_{n} \cos \left(\frac{n \pi x^{\prime}}{L}\right)+\sum_{n=1}^{\infty} b_{n} \sin \left(\frac{n \pi x^{\prime}}{L}\right)
$$

where

$$
\begin{aligned}
& a_{0}=\frac{1}{L} \int_{0}^{2 L} f\left(x^{\prime}\right) d x \\
& a_{n}=\frac{1}{L} \int_{0}^{2 L} f\left(x^{\prime}\right) \cos \left(\frac{n \pi x^{\prime}}{L}\right) d x \text { and } \\
& b_{n}=\frac{1}{L} \int_{0}^{2 L} f\left(x^{\prime}\right) \sin \left(\frac{n \pi x^{\prime}}{L}\right) d x .
\end{aligned}
$$

A periodogram which plots those frequency components with the greatest intensity or amplitude against the period shows which components bear significant meaning and which components are random 'noise'. In cyclical data, it may be found that a few frequencies are able to model the behaviour of the series relatively accurately. The low amplitude, noise frequencies may be discarded and a new, 'cleaner' time-series - free of noise and comprising only time-series signals characterised by the dominant frequencies - may thus be constructed.

\section{RESULTS AND DISCUSSION}

\subsection{Results}

\section{Cycle Frequency Identification}

The discrete Fourier transform assumes that the input signal (in this case, the nominal GDP) is statistically stationary, i.e. it has a constant mean through time. If the data were taken as is (due to the convex growth curve), considerably more weight would be given to more recent fluctuations as the scale has increased substantially in later years (2000s onwards), relative to the initial years (pre 2000s) - as shown in Figure 1. This would not present an accurate representation of the time series and the Fourier analysis would not identify cycles effectively. 
Figure 1. Nominal GDP in ZAR trillions, seasonally adjusted.

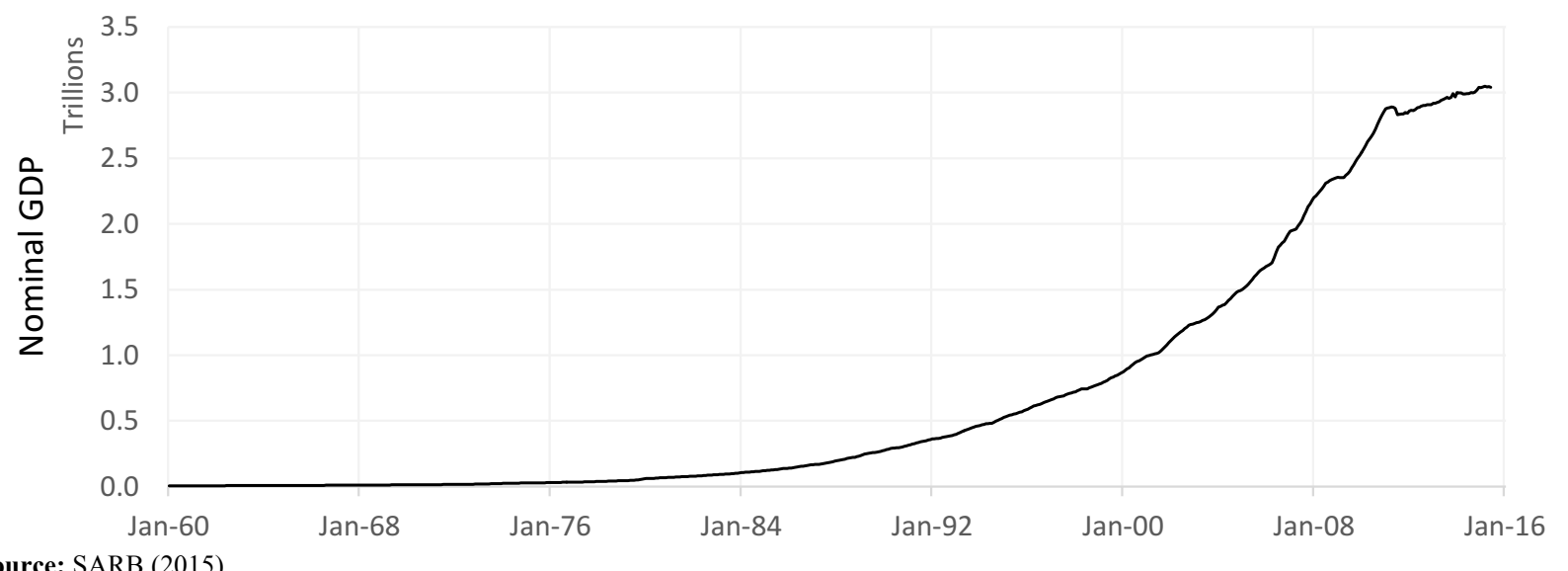

Source: SARB (2015).

An ADF test was performed shown in Table 1(a) and (b) to establish whether the data were non-stationary.

Table 1 (a). Augmented Dickey-Fuller test for stationarity: monthly GDP levels.

\begin{tabular}{|c|c|c|c|c|}
\hline Stationary test & \multicolumn{4}{|c|}{$5.0 \%$} \\
\hline Test & Stat & $p$-value & Critical value & Stationary? \\
\hline No Const & -2.2 & $2.5 \%$ & -1.9 & FALSE \\
\hline Const-Only & -5.8 & $0.1 \%$ & -2.9 & FALSE \\
\hline Const + Trend & -7.1 & $0.0 \%$ & -1.6 & FALSE \\
\hline${\text { Const }+ \text { Trend }+ \text { Trend }^{\wedge} 2}$ & -7.4 & $0.0 \%$ & -1.6 & FALSE \\
\hline
\end{tabular}

Table 1 (b). Augmented Dickey-Fuller test for stationarity: monthly GDP returns.

\begin{tabular}{|c|c|c|c|c|}
\hline Stationary test & \multicolumn{4}{|c|}{$5.0 \%$} \\
\hline Test & Stat & $p$-value & Critical value & Stationary? \\
\hline No Const & -2.2 & $2.5 \%$ & -1.9 & TRUE \\
\hline Const-Only & -5.8 & $0.1 \%$ & -2.9 & TRUE \\
\hline Const + Trend & -7.1 & $0.0 \%$ & -1.6 & TRUE \\
\hline Const + Trend + Trend $^{\wedge} 2$ & -7.4 & $0.0 \%$ & -1.6 & TRUE \\
\hline
\end{tabular}

The ADF test examines the inputs for the existence of a unit-root in the context of a hypothesis test. If a unit-root exists, we reject the null hypothesis and accept the alternative hypothesis that this root exists. The results showed that our assumption was correct and that we needed to perform a transformation to stationarise the data.

To stationarise the data, the natural logarithm difference from month to month was calculated to produce the percentage returns series shown in Figure 2. These returns do not scale with time and have a non-trending mean, so these are suitable for use in the Fourier analysis framework. 
Figure 2. De-trended GDP returns series using first differences.

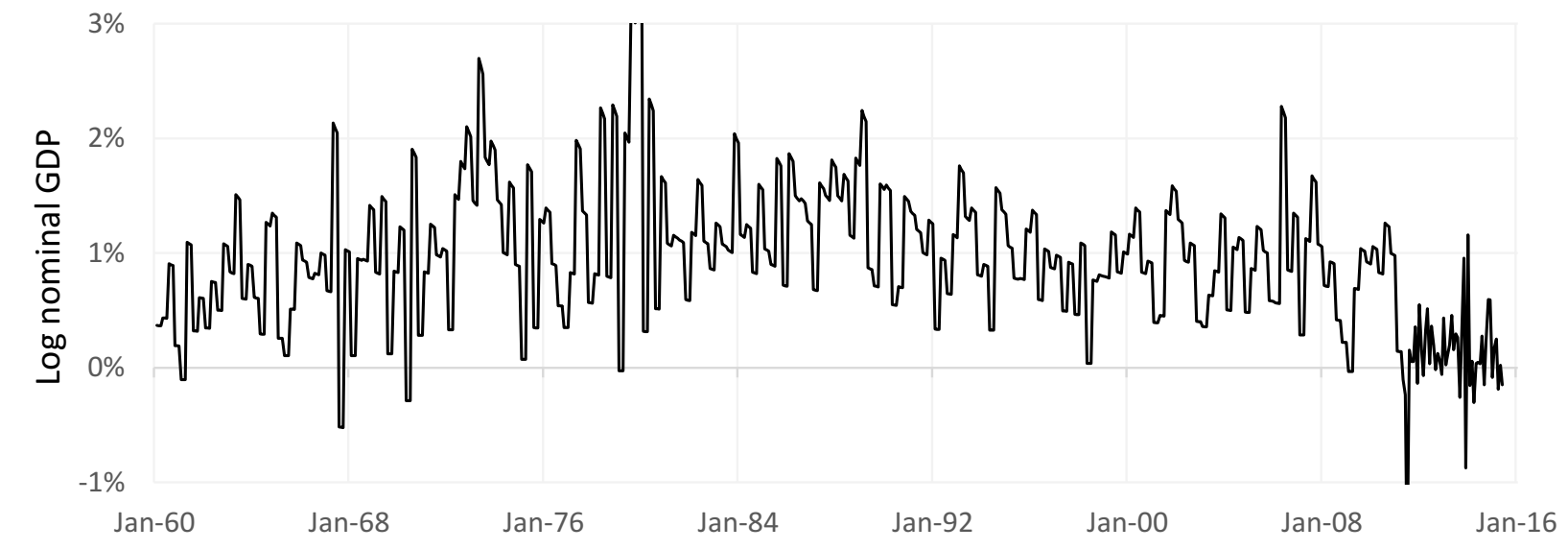

Source: Author calculations.

In the case of returns over the sample period, the mean monthly return is $1.05 \%$. This positive average produces the upward 'trend' observed in the monthly GDP level in Figure 1. We are interested in identifying the cyclical changes around this trend.

Because of the volatility in the returns series, we apply two filtering methods which extract the trend and cycle components from the series and produce a smoother returns series. These are the HP and BK filters. The log returns series is plotted alongside the filtered series in Figure 3. Summary statistics illustrating the effectiveness of the filters in capturing the trend and filtering through the noise appears in Table 2.

Figure 3. HP and BK-filtered log monthly nominal GDP (grey line).

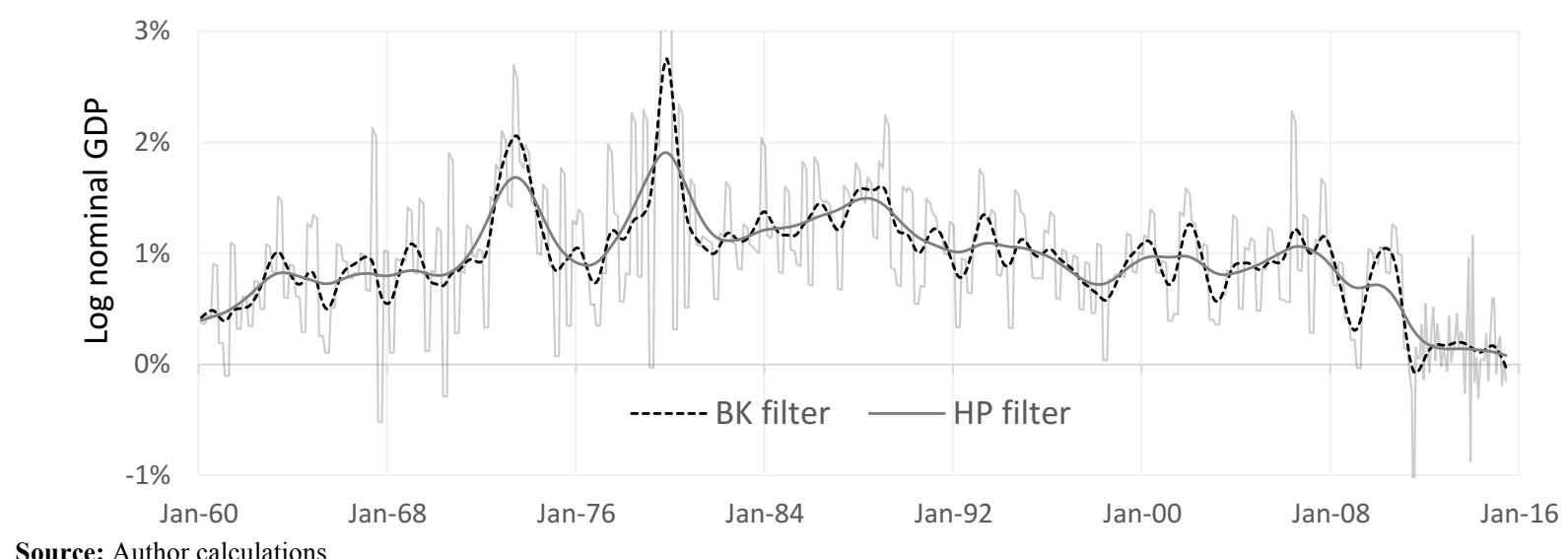

Table 2. Summary statistics illustrating the effects of filtering.

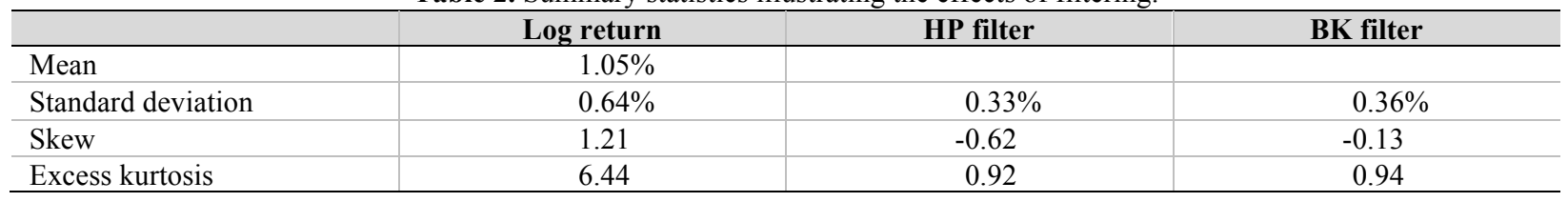


The standard deviation of the log returns series is $0.64 \%$, whilst the HP and BK filtered series produce less variation with standard deviations of $0.33 \%$ and $0.36 \%$ respectively. The filtered data also contain less excess kurtosis than the unfiltered returns series while there is a positive skew in the unfiltered series and negative skew of less magnitude in the filtered series. A Jarque-Bera normality test (Table 3) confirmed that the data were not normally distributed.

Table 3. Jarque-Bera test of normality on monthly returns at a significance level of 5\%.

\begin{tabular}{ccccc}
\hline Normality test & Z-Score & Critical Value & $\boldsymbol{p}$-Value & Pass? \\
\hline Jarque-Bera & 988.90 & 5.99 & $0.0 \%$ & FALSE \\
\hline
\end{tabular}

Severe excess-kurtosis and a positive skew, shown in a histogram plot with a normal distribution curve for comparison in Figure 4 below, characterise the monthly GDP returns series.

Figure 4. Frequency distribution of log returns series compared with the normal distribution curve.

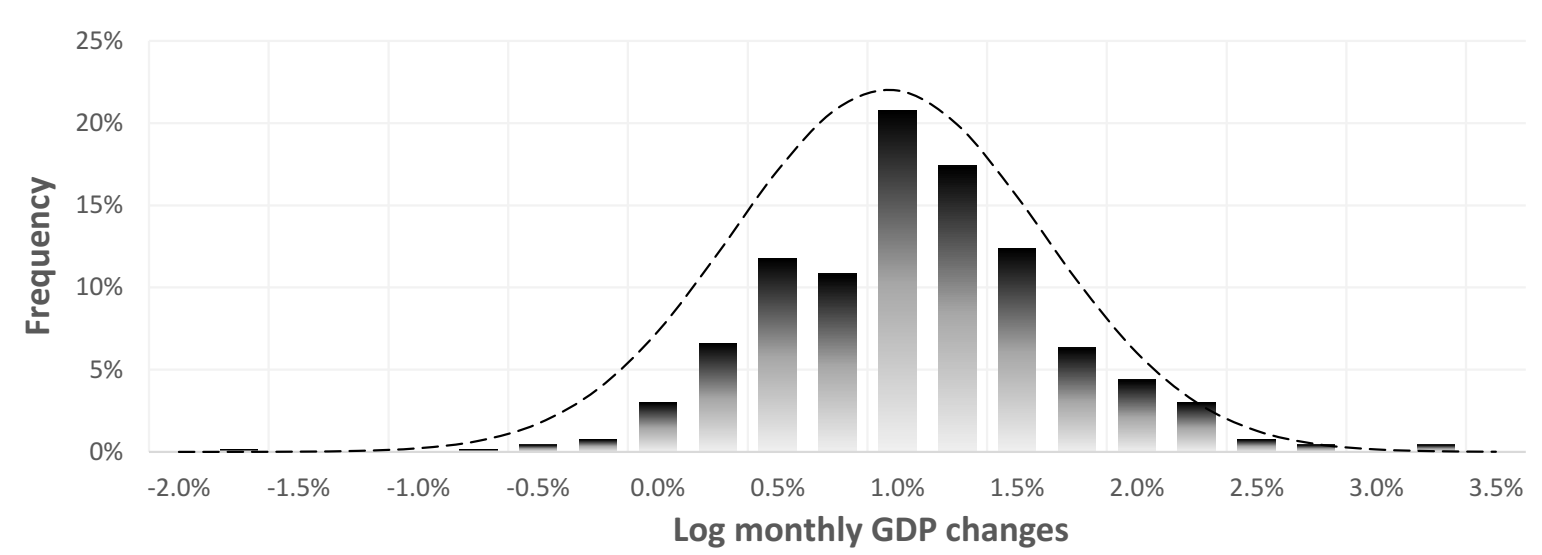

Source: Author calculations.

\section{Fourier Analysis}

Using the discrete version of the Fourier transform, the time-series of GDP returns was transformed from a timedomain representation into a frequency-domain representation. The time-series is decomposed into a series of sine and cosine waves occurring at different frequencies with different intensities, ${ }^{3}$ which in summation are able to exactly mimic the behaviour of the original signal. The power or amplitude of each frequency component (which explains the importance of the particular frequency in making up the original signal) is plotted against its period in Figure 5 below. The frequency is defined as $\frac{1}{\text { cycle (in months) }}$. The filtered series (Figure 5(b) and (c)) show much less noise than the unfiltered series as they rid the data of random deviations or 'noise.' The periodogram omits periods longer than 180 months, which distorts the analysis.

\footnotetext{
${ }^{3}$ Intensity, power and amplitude are used inter-changeably.
} 
Figure 5. Periodograms of (a) transformed log monthly return data, (b) the HP filtered log monthly returns and (c) BK filtered log monthly returns.
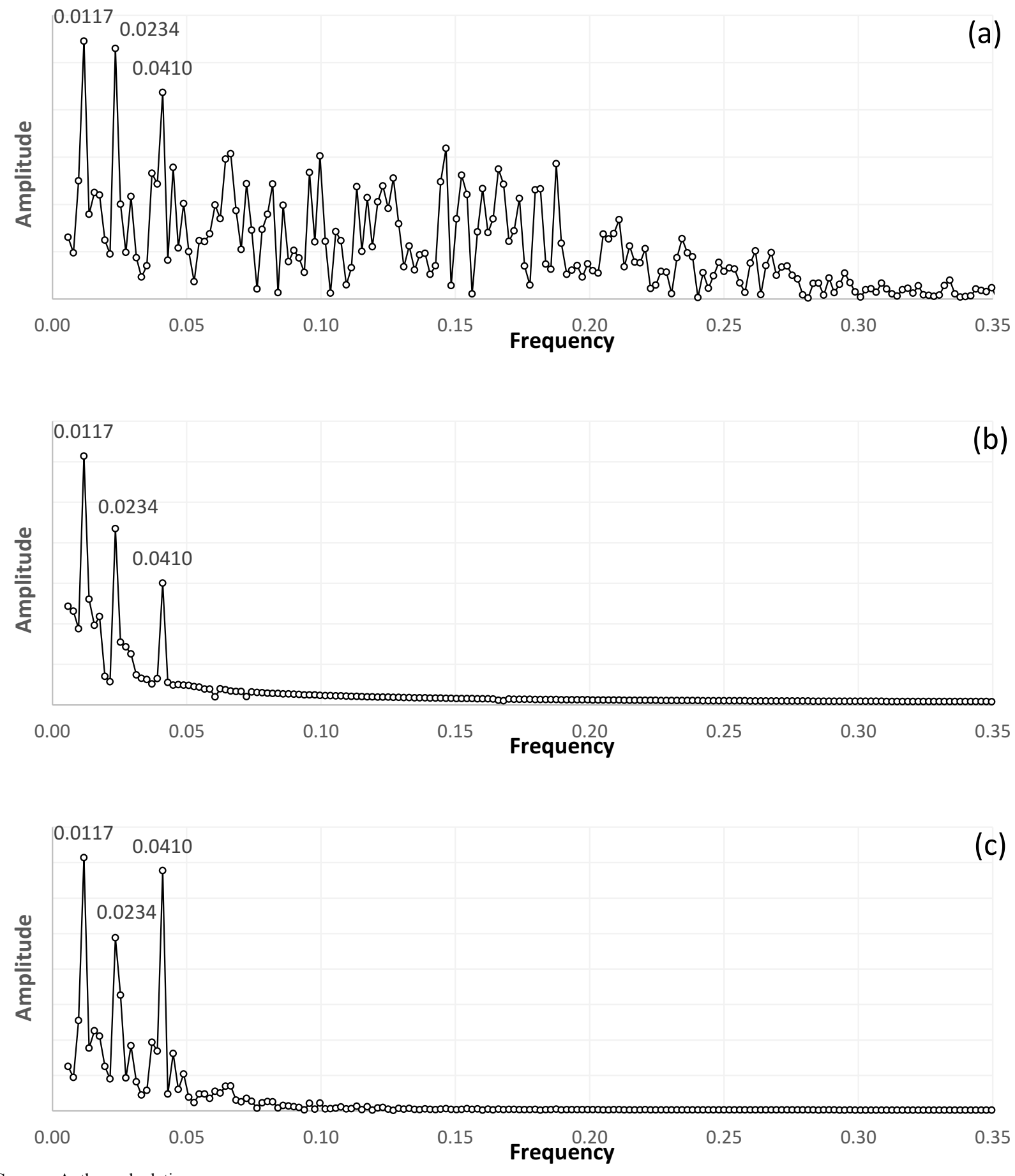

Source: Author calculations.

Three dominant frequencies are evident: these are at frequencies of 0.0117 (85.3 months for a full cycle or one full cycle every 7.1 years), 0.0234 ( 42.7 months for a full cycle or one full cycle every 3.5 years) and 0.0410 (24.4 months for a full cycle or one full cycle every 2.0 years). 
Botha (2004) found evidence of a 7.0 year cycle in South African GDP data using smooth transition autoregressive technique. This result confirms the result obtained above - that the dominant cycle present in South African GDP data using the Fourier transform is 7.1 years. The prominent cycle at 3.5 years is likely to be a harmonic of the 7.1 year cycle, so this cycle contains no economic information. The final significant cycle of 2.0 years is the South African business cycle (Gauteng Province, 2012).

\section{EMD}

The EMD was used to confirm cycle frequencies detected by the Fourier approach.

The process described in Section 3.2 (making data series stationary) is repeated until the local mean of $x(t)=0$ everywhere (within some pre-set tolerance). The resulting function is the first intrinsic model function (IMF). This IMF is subtracted from the original function and the entire process repeated on the remainder function to obtain the second IMF. This is repeated until all IMFs have been recovered and the remainder does not have sufficient local extrema to continue: this remainder is the trend.

The EMD technique was applied to the log monthly returns of nominal GDP over several iterations, and the results are shown in Figure 6.

Figure 6. EMD for log monthly nominal GDP after (a) one, (b) three and (c) six iterations. Note that all graphs are on the same timescales, but different y-axis scales.
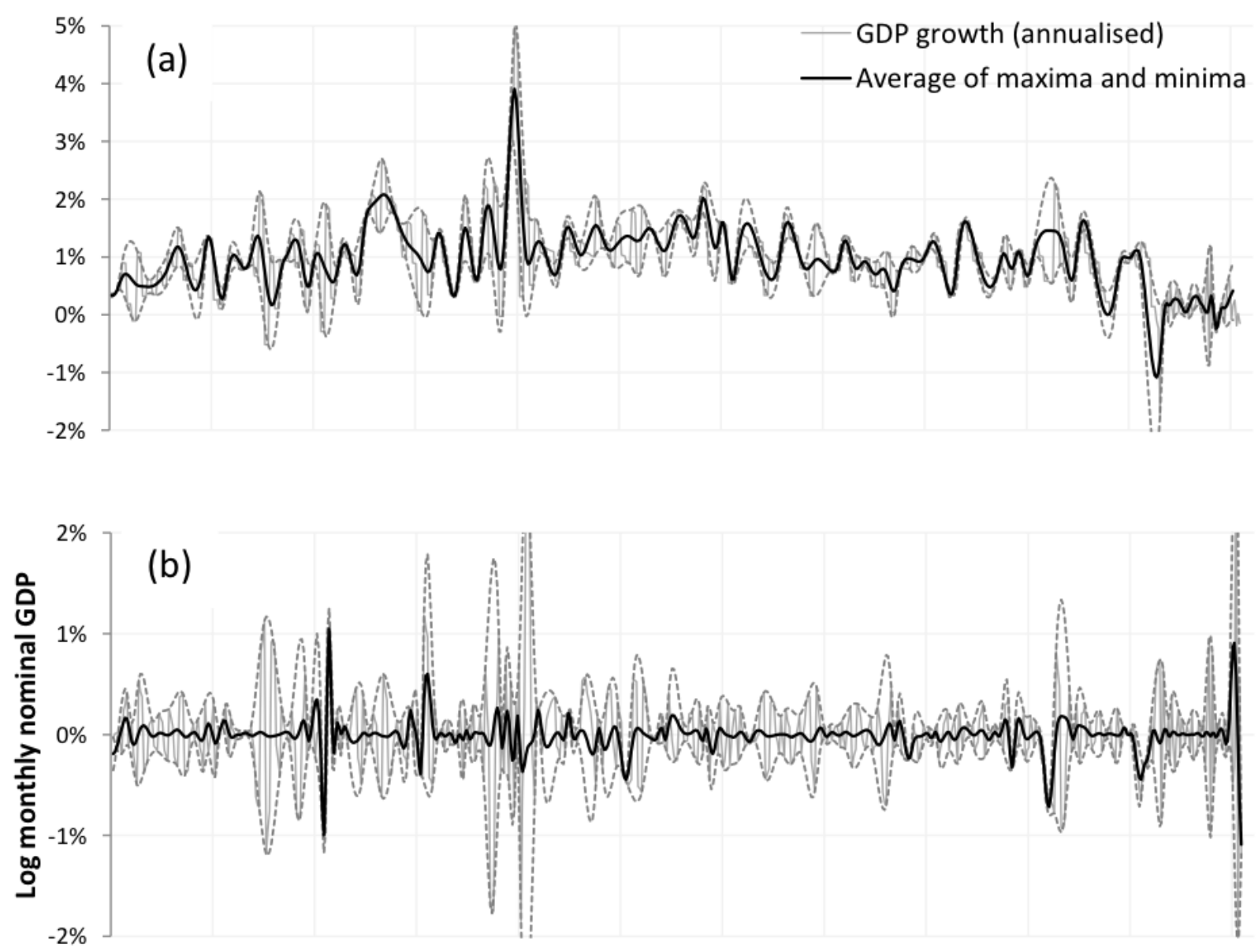

(Figure 6 continued on next page) 
(Figure 6 continued)

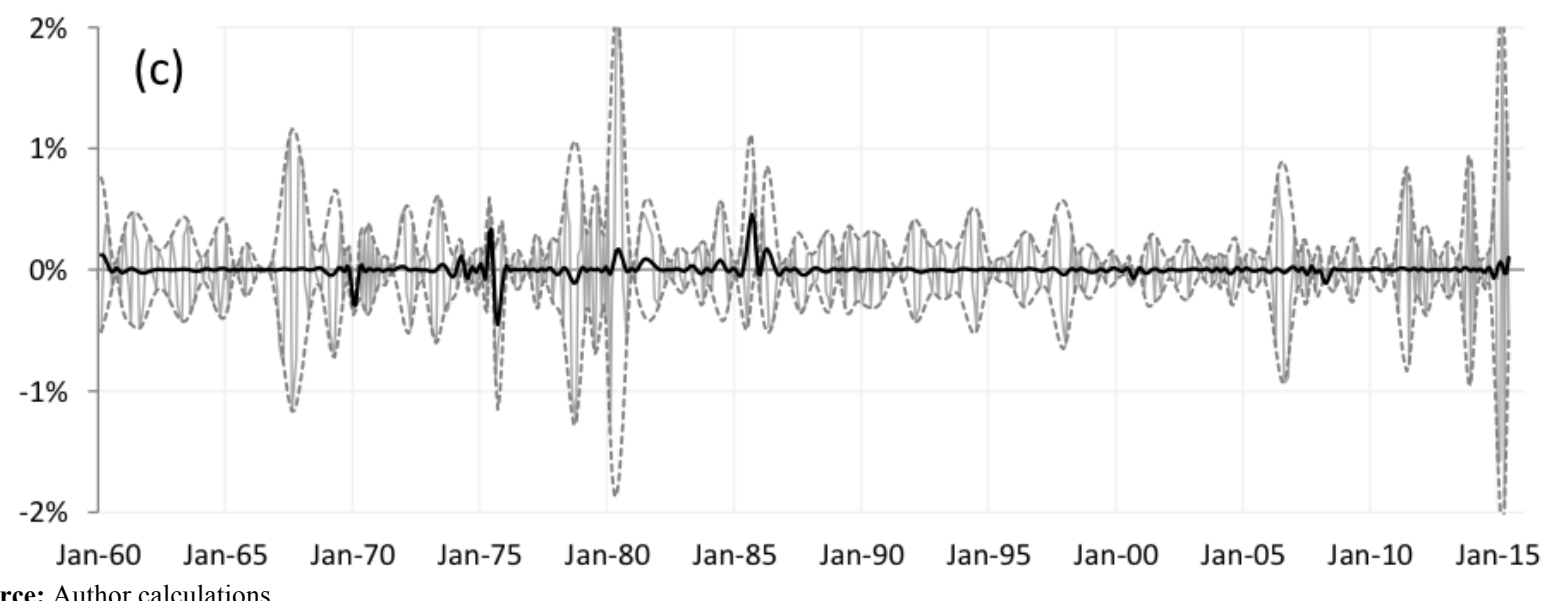

Source: Author calculations.

Spectral analysis of the IMFs gave the spectral decomposition as shown in Figure 7.

Figure 7: Spectral decomposition of EMD IMFs showing the same three dominant frequencies as those observed for the Fourier analysis.

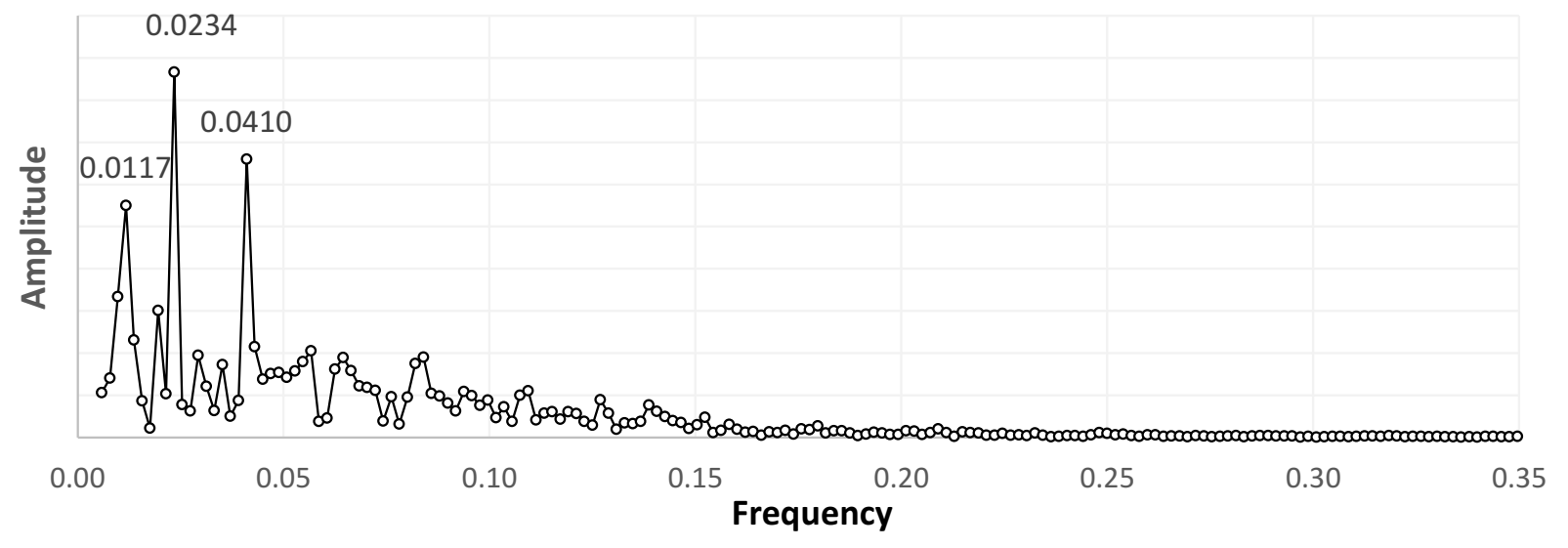

The same frequencies as those found by Fourier analysis were again dominant, i.e. cycles of 7.1 years, 3.5 years and 2.0 years. The confirmation by another mathematical technique provides some assurance that Fourier analysis is indeed identifying the correct underlying cycles present in the data.

\section{Forecast Potential}

Using the three dominant frequencies observed in the GDP dataset, a 3.5 year forecast was generated. This forecast used roughly 32 years of monthly data (i.e. from 1969 to 2012). The out-of-sample forecast of the log monthly nominal GDP was compared with historical data until mid-year 2015.

Figure 8 shows the 3.5 year log monthly GDP forecast from January 2012 to July 2015 using a combination of cycles generated from the three principal frequency components. The entire range of historical data was not plotted - only the last 16 years, since January 2005. 
Figure 8: Historical log monthly GDP and associated (fitted) Fourier cycles using frequencies from the dominant three cycles present in the data. Also shown is the forecast (out of sample prediction) of log monthly GDP from January 2012 to July 2015 and actual [realised] log monthly GDP in circles. One standard deviation above and below the log monthly GDP forecast is also shown (dotted lines).

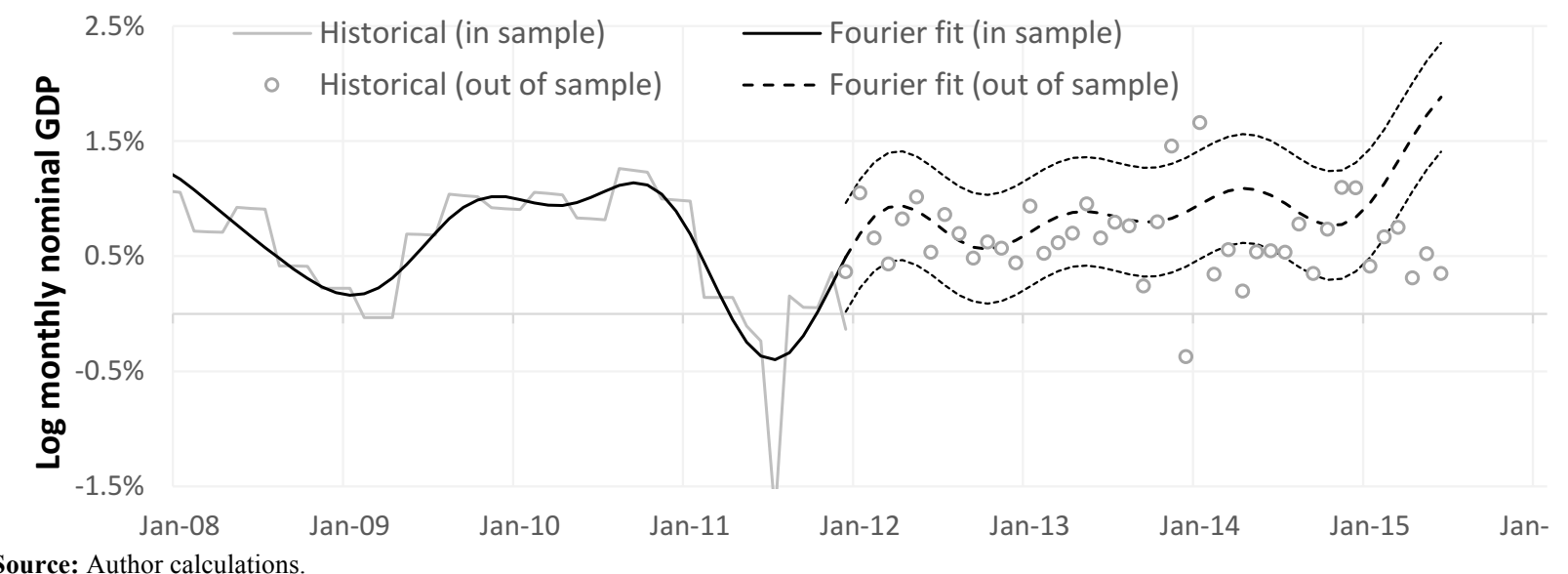

Source: Author calculations.

The forecast estimates using only three cycles forecast the future GDP reasonably accurately, although the uncertainty and negative GDP growth around January 2014 - which coincided with the Marikana unrest (Twala, 2014) - could not have been forecast and skewed the results.

\section{DISCUSSION}

Spectral analysis provides an alternative method of, in this case, economic cycle analysis, to the traditional method used by the SARB and Venter (2005) of micro and macro-economic indicators which lead, coincide with and lag the economy. The results of this study indicate that, using 665 months of GDP data, a significant cycle exists in the log monthly South African nominal GDP with a period of 7.11 years over the period January 1969 to July 2015 . Other prominent cycles are one of 3.5 years (possibly a harmonic of the 7.1 year cycle) and one at roughly 2.0 years (a possible South African business cycle).

These values were calculated using a Fourier transform methodology and these outputs were confirmed using EMD analysis.

Viewing the time series as comprising a general trend, a cyclical and an error component, the behaviour of each of these may be investigated in a similar way to Omekara (2013) who assessed each component separately before reassembling them to generate a forecast equation. Specifically, Omekara (2013) estimated the error component by evaluating the autocorrelation function of the residual for randomness. If the residual were not random, as in Omekara's (2013) case, a first order autoregressive model may be fitted to the error values. The BK filter separates a time-series into three components, but it is critical to use the appropriate parameters in the filter to produce meaningful results. Further studies may be able to establish these parameters and use the BK-filtered data to run Fourier analysis. If serial autocorrelation is present in the error component and can be taken into account via an autoregressive model as part of the forecast equation, forecast accuracy may improve.

The combination of cycles - which constitute the out of sample prediction of log monthly nominal GDP - are reasonably accurate in determining the possible future course of South African GDP - even out to 3.5 years from the forecast date. The majority of data (at a 95\% confidence level) were within one standard error of the forecast mean.

Fourier analysis does not identify the start and end points of a cycle. Liu et al. (2012) contend that with the length of a cycle identified, one can infer the start and end points. This is the reverse of the method used in Bry \& Boschan (1971), in which the length of a cycle is inferred by first identifying the start and end points. 
The phases of the various (three) components used to generate Figure 8 provide turning point dates and business cycle phase changes (also demonstrated by Botha, 2004). These phases - which interact and interfere via superposition may be identified and isolated and the maxima and minima of each cycle explored. Knowledge of these points could be of considerable importance to policy makers who may wish to be forewarned of economic slowdowns or upturns in order to manage expectations and reign in profligate lending, for example.

\section{CONCLUSION AND RECOMMENDATIONS}

\subsection{Conclusion}

Using Fourier and periodogram analysis to study South African GDP from January 1969 to December 2011, clear individual cycles were isolated and identified, lasting 7.1 years, 3.5 years and 2.0 year respectively. This result, though obtained using an alternative quantitative approach, compares favourably with the result of Botha (2004), who found a 7.0 year cycle in quarterly South African GDP from 1961 to 2003. A simple log transformation allows the use of Fourier analysis methods and that filtering using the HP and BK filters helps omit the majority of random 'noise' whilst still preserving the integrity of explanatory cycles present in the data.

Forecasting future GDP, even out to 3.5 years from the analysis period and using the three frequency components showed good agreement with realised log monthly nominal GDP values. The results using the Fourier transform are encouraging and have demonstrated that the technique has potential for forecasting GDP. Non-obvious cyclical patterns in the time do-main data are considerably clearer in the frequency domain.

\subsection{Recommendations for Further Study}

\section{Business Cycle Data Representation}

Nominal GDP was used as a proxy for the SA business cycle. GDP growth is considered the most natural indicator of an economy's aggregate business cycle by the Basel Committee for Banking Supervision (BCBS, 2010). However, a number of macroeconomic indicators with useful business cycle information could potentially have been used. These include aggregate real credit growth, the credit-to-real GDP growth ratio and the leading, lagging and co-incident indicators used by the SARB to measure the business cycle. The BCBS considers real credit growth as a natural measure of supply (since boom periods leading to a peak in the business cycle are characterized by rapid credit expansion and credit contraction has typically heralded credit crunches (BCBS, 2010). For a comprehensive study of the use of Fourier series methods in detecting the length and timing of South African business cycles, the above data should also be analysed.

\section{Data Selection}

Monthly GDP data showed substantial variation. Quarterly data combined with weighted moving averages and filtering would help to smooth volatility in the short term and could be considered in future studies. In South Africa's case, the recommendation is that 256 quarters ( 768 months) are used to ensure the reliability of the results, with the caveat that economic, environmental and political factors may have changed considerably over this period.

\section{Return Calculation}

Returns were calculated by taking the natural logarithms of month-to-month GDP levels and not month-on-month returns. This approach produces monthly changes with less autocorrelation than using month-on-month data, which gives an annual change. Other methods of return calculation could be attempted.

\section{Power Spectrum Estimation}

Use was made of the non-parametric, periodogram method to calculate the power of a frequency component. Masset (2008) compared the parametric methods of Yule-Walker and Burg (Tjostheim \& Paulsen, 1983) and the nonparametric methods of the periodogram and Welch method (Welch, 1967). The non-parametric methods was 
accompanied by more 'noise' over the spectrum and the difference between the periodogram and Welch method was considerably greater than the difference in the parametric methods. Preceding Fourier analysis, one should ensure the most suitable method to estimate the power spectrum is used or provide a comparison of the results before further analysis.

\section{Forecasting Issues - Modelling Time Series Components}

This paper made use of unfiltered returns data as the Fourier tool used was able to separate the cyclical components from the general trend internally. However, using the BK filtered returns or any method that can separate the trend, cycle and error components of a time series will allow future studies to attempt to model the error component identified if it is not random, as in Omekara (2013). This may improve the accuracy of forecasts.

\section{Forecasting Issues - Determining Cycles Phases}

The methods used to determine the length of the SA business cycle provided satisfactory results in this paper, but ascertaining the approximate current position of the economy on the business cycle was not addressed in full. For this purpose, further studies should include Fourier analysis and forecasting of various measures of the business cycle, not only the rate of GDP growth. Phase differences between leading, lagging and co-incident cycles may provide valuable information to pin-point the current position of the economy and a composite cycle of such indicators may provide a more accurate forecast.

\section{Statistical Measurement of Forecast Accuracy}

The forecasts in this paper were not tested by any statistical measure of significance and thus cannot be compared to other forecast tools. Although testing may become rigorous, at a minimum, testing for correlation and performing a simple regression to predict future GDP returns based on the path of the reconstructed signal is proposed. A multiple regression hypothesis test may yield helpful results regarding the significance of each frequency component in predicting future values. The periodogram performed this function by ordering components according to amplitude, but a multiple regression could prove this statistically.

\section{Wavelets}

Wavelets are relatively new tools in economics and finance. These provide an attractive way of analysing financial datasets as they are able to represent data series from both the time and frequency perspectives simultaneously (Scargle, 1993). Hence, they permit the breakdown of market activity into different frequency components and the study of these dynamic components separately (Daubechies, 1988). Wavelets do not suffer from some of the limitations of standard frequency-domain methods, like Fourier analysis used in this paper, and can be employed to study a financial variable, whose evolution through time is dictated by the interaction of a variety of different frequency components (Vetterli \& Herley, 1992). These components may behave according to non-trivial (noncyclical) dynamics - e.g., regime shifts, jumps, long-term trends (Masset, 2008). ${ }^{4}$ Due to the complex nature and presence of such non-trivial dynamics in South African GDP data under study, using wavelet analysis on South African and other similar GDP datasets which are characterised by non-cyclical dynamics is strongly encouraged.

\section{AUTHOR BIOGRAPHIES}

Daniel Thomson obtained his Bachelor of Commerce degree at the University of Stellenbosch and his Honours degree in Financial Analysis and Portfolio Management from the University of Cape Town, South Africa. In mid-2015, Daniel transferred to the UK where he is now a fixed income and equity risk analyst for Aviva Investors, London. He is also enrolled at North West University, South Africa, where he is undergoing his postgraduate studies in risk management. Daniel's Masters dissertation will focus on the role of forecasting in finance.

\footnotetext{
${ }^{4}$ For a non-technical introduction to wavelets and their benefits compared to pure-frequency domain analysis, see Masset (2008). 
Gary van Vuuren obtained his PhD in nuclear physics from the University of Natal, South Africa and then transferred to financial risk management where he completed a $\mathrm{PhD}$ in credit risk management in 2005 . He transferred to the UK in 2003 and has worked in retail banks, asset managers, investment banks, financial consultancies and credit rating agencies. He currently works for Aviva Investors, London, in the model validation group. He is also an extraordinary professor at North West University, South Africa.

\section{REFERENCES}

Aron, J., and Muellbauer, J. 2002. Evidence from a GDP Forecasting Model For South Africa IMF Staff Papers, 49, IMF Annual Research Conference, 2002: 185-213.

Baxter, M., \& King, R. G. 1999. Measuring business cycles: approximate band-pass filters for economic time series. The Review of Economics and Statistics, 81(4): 575-593.

BCBS. 2010. Guidance for national authorities operating the countercyclical capital buffer. Bank for International Settlements. Retrieved from: http://www.bis.org/publ/bcbs187.pdf [accessed 2015-06-19].

Bosch, A., and Ruch, F. 2013. An alternative business cycle dating procedure for South Africa. South African Journal of Economics, 81(3): 491-516.

Bry, G., \& Boschan, C. 1971. Cyclical analysis of time series: selected procedures and com-puter programs. New York: Columbia University Press.

Canova, F. 1994. Detrending and turning points. European Economic Review, 38(3-4): 614-623.

Canova, F. 1998. Detrending the business cycle. Journal of Monetary Economics, 41(2): 475-512.

Chevillon, G. 2009. Multi-step forecasting in emerging economies: An investigation of the South African GDP. International Journal of Forecasting, 25(3): 602-628.

Cody, M. A. 1994. The wavelet packet transform. Dr. Dobb's Journal, 19(4): 44-46 and 50-54.

Cogley, T., \& Nason, J. M. 1995. Effects of the Hodrick-Prescott Filter on trend and difference stationary time series: implications for business cycle research. Journal of Economic Dynamics and Control, 19(1): 253-278.

Cooley, J. W. \& Tukey, J. W. 1965. An algorithm for the machine calculation of complex Fourier series. Mathematics of Computation, 19(6): 297-301.

Cunnyngham, J. 1963. Spectral analysis of time series. Working Paper No. 16, U.S. Department of Commerce, Bureau of Census, New York.

Daubechies, I. 1988. Orthonormal bases of compactly supported wavelets. Communications in Pure Applied Mathematics, 41(7): 906-966.

Debnath, L. 2011. A short biography of Joseph Fourier and historical development of Fourier series and Fourier transforms. International Journal of Mathematical Education in Science and Technology, 43(5): 1-24.

Gauteng Province. 2012. Business cycles and their impact on the South African economy. Quaterly Bulletin: Provincial Treasury: Republic of South Africa. October to December 2012.

Granger, C. W. J., \& Morgenstern, J. 1964. Spectral analysis of stock market prices. International Review for Social Sciences, 16(1): $1-27$.

Granger, C. W. J. 1966. The typical spectral shape of an economic variable. Econometrica, 34(1): 150-161.

Guay, A., and St-Amant, P. 2005. Do the Hodrick-Prescott and Baxter-King filters provide a good approximation of business cycles? Annales d'Economie et de Statistique, 77(Jan - March): 133-155.

Hamilton, J. D. 1994. Time Series Analysis, Princeton.

Harvey, A. C., \& Jaeger, A. 1993. Detrending, stylized facts and the business cycle. Journal of Applied Econometrics, 8(3): 231247.

Hodrick, R. J., \& Prescott, E. C. 1997. Postwar U.S. business cycles: an empirical investigation. Journal of Money, Credit, and Banking, 29(1): 1-16.

Huang, N. E. Shen, Z., Long, S. R., Wu, M. C., Shih, E. H., Zheng, Q., Tung, C. C. and Liu, H. H. 1998. The Empirical mode decomposition method and the Hilbert spectrum for non-stationary time series analysis, Proceedings of the Royal Society of London, A454(2): 903-995.

Iacobucci, A. 2003. Spectral analysis for economic time series, Documents de Travail de I'OFCE.

King, R. G., \& Rebelo, S. T. 1993. Low frequency filtering and real business cycles. Journal of Economic Dynamics and Control, 17(1-2): 207-231.

Lindgren, G., Rootzén, H and Sandsten, M. 2013. Stationary stochastic processes for scientists and engineers. Chapman and Hall, USA, 200p.

Liu, L., Paki, E., Stonehouse, J., and You, J. 2012. Cycle Identification: An old approach to (relatively) new statistics. NZAE Annual Conference, Statistics New Zealand.

Masset, P. (2008). Analysis of financial time-series using Fourier and wavelet methods, Faculty of Economics and Social Science, (October), pp.1-36. Retrieved from: https://psutn2009.googlecode.com/svn-history/r5/trunk/SSRNid1289420.pdf

Nerlove, M. 1964. Spectral analysis of seasonal adjustment procedures. Econometrica, 32(2): 241-286. 
Omekara, C. O., Ekpenyong, E., J., and Ekrete, M., P. 2014. Modelling the Nigerian inflation rates using periodogram and Fourier series analysis. Lagos: Nigerian Central Bank.

Plessis, S., Smit, B. \& Steinbach, R. 2014. South African Reserve Bank Working Paper Series A medium-sized open economy DSGE model of South Africa, (July).

Praetz, P. D. 1973. A spectral analysis of Australian share prices. Australian Economic Pa-pers, 12(5): 70-78.

Ravn, M. O., \& Uhlig, H. 2002. On adjusting the Hodrick Prescott filter for the frequency of observations. The Review of Economics and Statistics, 84(2): 371-375.

SARB - South African Rerserve Bank. 2015. Retrieved from: http://wwwrs.resbank.co.za/webindicators/SDDSDetail.aspx?DataItem=NRI6006D

Scargle J., Steiman-Cameron, T., Young, K., Donoho, D. L., Crutchfield, J. P. and Imamura, J. 1993. The quasi-periodic oscillations and very low frequency noise of Scorpius X-1 as transient chaos: a dripping handrail? Astrophysical Journal, 411(5): L91-L94.

Telser, L.G., Granger, C. W. J. \& Hatanaka, M. 1967. Spectral analysis of economic time series. Journal of the American Statistical Association, 62(3). Retrieved from: http://www.jstor.org/stable/2283996?origin=crossref.

Tjostheim, D. \& Paulsen, J. 1983. Bias of some commonly-used time series estimates. Biometrika, 70(2): 389-399.

Twala, C. 2014. The Marikana massacre: a historical overview of the labour unrest in the mining sector in South Africa. Southern African Peace and Security Studies, 1(2): 61-67.

van Vuuren, G. 2012. Basel III Countercyclical capital rules: implications for South Africa. South African Journal of Economic and Management Sciences, 15(3): 76 - 89.

Venter, J. C. 2005. Reference turning points in the South African business cycle: recent developments. SARB Quarterly Bulletin, September, 61-70. Retrieved from: http://www.esaf.org/internet/Publication.nsf/LADV/EE79538CB86F96DA42257083004E8875/\$File/ART092005.pdf nhttp://www.oecd.org/dataoecd/54/26/34898202.pdf

Vetterli, M. \& Herley, C. 1992. Wavelets and filter banks: theory and design. IEEE Transactions on Signal Processing, 40(2): 2207-2232.

Welch, P. D. 1967. The use of fast Fourier transform for the estimation of power spectra: a method based on time averaging over short, modified periodograms. Transistor, Audio and Electroacoustics, AU-15(1): 70-73. 